\title{
RESEARCH ON STRATEGIC ARCHITECTURE OF COLLABORATIVE BUSINESS SYSTEM
}

\author{
Zhiwei Mi ${ }^{1}$, Wenwu $\mathrm{Mao}^{2}$, Minglun Fang ${ }^{1}$ \\ ${ }^{\prime}$ CIMS \& Robot Center of Shanghai University, Shanghai University, China; Email: \\ zwmi@mail.shu.edu.cn. ${ }^{2}$ College of Engieering, Shanghai Fisheries University, China.
}

\begin{abstract}
Collaborative business system is perfect combination of the best business practices with information technology. Collaborative business system is an innovation and enabled changes to traditional rules for doing business. The aim of this paper is: firstly to provide an overview of collaborative business by outlining the essence and the framework; secondly, to put forward to the architecture of collaborative layer, the most important part of the framework.
\end{abstract}

Key words: Collaborative business system, theoretical framework.

\section{INTRODUCTION}

Even in today's New Economy, traditional rules for doing business still hold true, but the Internet has changed the way business works, linking manufacturers, suppliers, distributors, customers, partners, and employees across entire sectors, by establishing collaborative business networks (virtual communities). Creating value by collaboration, empowerment, and integration in the Internet economy depends on the ability of businesses to work together with its customers, suppliers, and the like across traditional enterprise boundaries in virtual global networks.

The following evolution has recently taken place in the business world. Originally, companies were looking at cost reduction and efficiency by integrating internal business processes. So they use 'ERP functionality' to achieve this. ERP offer enterprise-centric functionality (management of Human Resource, Finance, Material) to integrate internal core processes.

Then, companies realized they must establish relationships with their suppliers and customers, to share information to reduce costs and time within their supply chain network. So the next generation of new dimension

This project is Supported by China Shanghai Municipal Education Commission under the grant No.03AK80.

Please use the following format when citing this chapter:

Mi, Zhiwei, Mao, Wenwu, Fang, Minglun, 2006, in International Federation for Information Processing (IFIP), Volume 207, Knowledge Enterprise: Intelligent Strategies In Product Design, Manufacturing, and Management, eds. K. Wang, Kovacs G., Wozny M., Fang M., (Boston: Springer), pp. 944-949. 
software products appeared, extending ERP, taking functionality out of the company, to bring value through cooperation along the supply chain.

Currently, the Internet has enabled a collaborative environment where value is created through collaboration within business communities. Collaborative business system builds on ERP functionality and also extends the following:

(1) Offering collaborative functions.

(2) Generating value through collaborative possibilities.

(3) Seamlessly integrating heterogeneous platforms.

The term of collaborative business was mainly introduced in 2000 by the world largest commercial software vendors, for instance, SAP, EDS, based on the combination of their best business practices with information technology. Since the introduction of the term, it has received growing interested and research both in literature as well as from industrial practice ${ }^{1 \sim 7}$.

The aims of this paper are to: firstly provide an overview of collaborative business system by outlining the essence and the framework; secondly explain in greater detail architecture of collaborative layer, the most important part of the framework.

\section{FRAMEWORK OF COLLABORATIVE BUSINESS SYSTEM}

So far, there is no formal and official definition of collaborative business system. The description of collaborative business system proposed here is only trying to extract the essence:

(1) Collaborative business system is complex system engineering. It regards business community as an entire system in which the parts are dynamic and interrelated, and as well business community and their environment are also regarded as a whole, enterprises should have social responsibilities.

(2) Collaborative business system is an innovation and enabling changes to traditional operating rules for doing business.

(3) Collaborative business system is synthetic approaches and tools using various theories, technologies and methodologies.

(4) Information technology enables collaborative business. Collaborative business system is perfect combination of the best business practices with information technology.

(5) Collaborative business is the task of creating value and benefits for each partner (so called win-win) through collaboration within business community with the aim of improving the competitiveness as a whole, as well fulfilling requirement of customers. 
(6) The collaboration and integration are critical in the collaborative business.

According to the description above, the paper puts forward to theoretical framework of collaborative business system as figure 1 . As you can see from the figure 1, the theoretical framework consists of three layers:

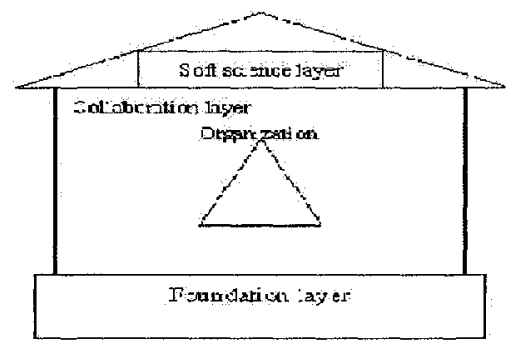

Figure 1. Theoretical framework of collaborative business system

(1) Foundation layer: this layer is the physical foundation of business community running, including various soft and hard resources, for example, distribution centers, ports, plant, other locations, transportation lane, material, tools, flexible manufacturing system, material handling system, computer; standards and protocols.

(2) Collaboration layer: this layer is the core of theoretical framework. This layer brings all together within business communities, coordinates the business operation, keep everything operating at peak efficiency. It consists of three parts, collaborative organization, collaborative business processes, integration and application platform. This layer will be described in great detail below.

(3) Soft science layer: this layer is comprehensive implementation solution available to streamline and facilitate collaborative business system. It consists of science theories, regulations, visions, methodologies, tools, services and training, etc.

\section{THE CORE OF THEORETICAL FRAMEWORK}

Compared with linear, sequential and separated business operation of traditional SCM solutions, the one of most important objectives of collaborative layer of theoretical framework is to transition today's fragmented sequential supply chain to synchronizing and coordinating and adaptive supply chain network.

The paper divides collaborative layer into three levels of collaboration. 


\subsection{Collaborative Organization}

One of the first steps in configuring the Collaborative business system is mapping and modeling the collaborative organizational structure in accordance with strategic business requirement of collaborative business using the organizational units.

From the business practices, organizational forms within collaborative organizational structure includes a wide array ranging from long-term purchasing agreements to co-marketing and licensing agreements, to $R \& D$ collaboration teams, to joint ventures. So, a collaborative organizational structure should create and build a close, medium-long term, mutually beneficial partnerships between two or more organizational units during all phases of business relationships. Resources, knowledge, and capabilities within collaborative organizational structure are shared with the objective of enhancing the competitive position of each unit. Centralization and decentralization are the two extremes in the collaborative organizational structure.

Despite the strategic alliances between enterprises, for example, are growing at ever increasing rate, many alliances fail with low success rates. Lack of communication and mistrust and even cheating are the main causes.

\subsection{Collaborative business processes}

Based on analysis of operational data from the transactional enterprise resources planning, collaborative business process supports continuously redesigning, coordination and optimization of business processes for gaining competitive advantage.

Collaborative business processes consists of the following contents as figure 2 .

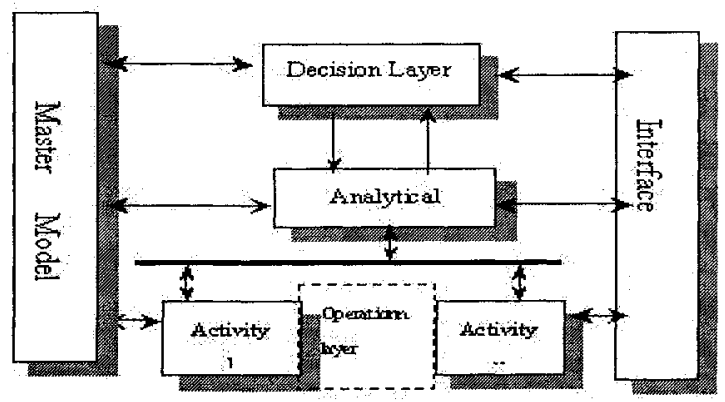

Figure 2. The Structure of Collaborative business 


\subsection{Integration and application platform}

The evolution of IT infrastructure from mainframe to client/server, to web service architecture reflects the ongoing adaptability and flexibility. Open, flexible IT infrastructure for collaboration across companies boundaries, e.g. built on the web service concept and environment, together with the integration of Internet, enables today's collaborative business system.

The paper considers the integration and application platform as the technical foundation for Collaborative business system. It delivers a complete, open and flexible infrastructure that easily integrate the three areas as belows:

(1) Integrate all participating people involved in the business process, whether within an enterprise or outside of it (internal and external, all the relevant roles).

(2) Manage all the relevant information (structured and unstructured) for the whole business processes through data warehouse and knowledge management, for example.

(3) Integrate all systems required for the business processes integration through integration technology, e.g. database oriented technologies, message oriented technologies, distributed object technologies, interfaced oriented technologies, etc, regardless of whether they are internal or external systems.

\section{CONCLUSION}

Collaborative business system is advanced management thoughts, methodologies and tools through which customers, suppliers, partners, employees, organizational units, business processes and information are brought together to form one collaborative unit within business community.

The foundation for achieving collaboration is as followings:

(1) Modern information and communication technology.

(2) Collaborative business processes, especially for advanced planning, optimization and controlling.

(3) Strategic alliance between organizational units.

\section{REFERENCES}

1. Escudero, L.F., Galindo, E., Garcia, G., Gomez, E., Sabau, V., 1999. Schumann, a modeling framework for supply chain management under uncertainty. European Journal of Operational Research 119, $14-34$. 
2. Fawcett, S.E., Myers, M.B., 2001. Product and employee development in advanced manufacturing: Implementation and impact. International Journal of Production Research $39,65-79$.

3. Fox, M.S., Barbuceanu, M., Teigen, R., 2000. Agent oriented supply chain management. International Journal of Flexible Manufacturing Systems 12, 165 - 188.

4. G. Gunnarsson, S. Jonsson, Charge the relationships and gain loyalty e.ects: Turning the supply link alert to IT opportunities, European Journal of Operational Research. 144 (2003) $257-269$.

5. G. Stefansson, Business-to-business data sharing: A source for integration of supply chains, International Journal of Production Economics 75 (2002) 135 - 146.

6. Cooper, M.C., Lambert, D.M., Pagh, J.D., 1997. Supply chain management: More than a new name for logistics. International Journal of Logistics Management 8 (1), 1 - 13. 